\title{
SABERES DEL DOCENTE Y REPRESENTACIONES SOCIALES: IMPLICANCIAS PARA LA ENSEÑANZA DE LAS CIENCIAS NATURALES
}

KNOWLEDGE OF THE TEACHER AND SOCIAL REPRESENTATIONS: IMPLICATIONS FOR TEACHING OF NATURAL SCIENCES

Volumen 11, Número 2

pp. 1-28

Este número se publicó el 30 de agosto de 2011

Susana Beatriz Aguilar Claudia Alejandra Mazzitelli

Monica Sonia Chacoma

Miriam Teresita Aparicio

La revista está indexada en los directorios:

LATINDEX, REDALYC, IRESIE, CLASE, DIALNET, DOAJ, E-REVIST@S,

La revista está incluida en los sitios:

REDIE, RINACE, OEI, MAESTROTECA, PREAL, HUASCARAN, $\underline{\text { CLASCO }}$ 


\title{
SABERES DEL DOCENTE Y REPRESENTACIONES SOCIALES: IMPLICANCIAS PARA LA ENSEÑANZA DE LAS CIENCIAS NATURALES KNOWLEDGE OF THE TEACHER AND SOCIAL REPRESENTATIONS: IMPLICATIONS FOR TEACHING OF NATURAL SCIENCES
}

\author{
Susana Beatriz Aguilar ${ }^{1}$ \\ Claudia Alejandra Mazzitelli2 \\ Monica Sonia Chacoma ${ }^{3}$ \\ Miriam Teresita Aparicio ${ }^{4}$
}

Resumen: En este artículo presentamos un análisis comparativo de tres estudios realizados con docentes de distintos niveles educativos de la provincia de San Juan (Argentina) y en los que, a través de técnicas de evocación y jerarquización de palabras, identificamos la estructura de sus representaciones sociales sobre distintos aspectos vinculados a la enseñanza y al aprendizaje de las Ciencias Naturales. Para analizar las estructuras e identificar los elementos comunes que atraviesan estos estudios, consideramos la clasificación de saberes del docente de Braslavsky y Birgin (sustantivo, pedagógico e institucional). Los resultados obtenidos nos han permitido acceder a información importante acerca de los significados compartidos por este grupo profesional. Así, la similitud entre las estructuras de los distintos grupos nos muestra que existe una representación que permite describir lo que son y lo que hacen los docentes, correspondiéndose con los postulados educativos tradicionales, marcando las diferencias con otros grupos y otras actividades profesionales. Las categorías que se presentan de modo constante, más allá de las temáticas planteadas en cada caso particular, se vinculan con el hecho de "educar", es decir, formar sujetos escolarizados para el mundo social en el cual se está inserto. De esta manera, podemos pensar que aunque las demandas sociales ejercen una fuerte presión sobre los docentes e intentan desviarlos en su desempeño profesional hacia otras funciones (asistenciales, sanitarias o de contención social), éstas no constituyen la esencia de la función social que ellos se auto-atribuyen y pretenden conservar a lo largo del tiempo.

\section{Palabras clave: SABERES DOCENTES; REPRESENTACIONES SOCIALES; CIENCIAS NATURALES, ARGENTINA}

\begin{abstract}
This article presents a comparative analysis of three studies made with teachers from different educational levels in the province of San Juan (Argentina), in which we identified the structure of their social representations - through techniques of evocation and hierarchical organization of terms - about different aspects related to the teaching and learning of Natural Sciences. To analyze the structures and identify the common elements that cut across these studies and present certain stability, we considered the classification of teacher's knowledge offered by Braslavsky and Birgin (substantive, pedagogic and institutional). The obtained results have allowed us to have access to important information about the meanings shared by this professional_group. Thus, the similarity between the structures of the different groups shows that there exists a representation that allows us to describe what these teachers are and what they do, which correspond to traditional education approaches, marking the differences with other groups and other professional activities. The categories that appear in a constant way, beyond the topics proposed in every particular case, are related to the fact of "educating", that is, developing schooled subjects for the social world in which they live. Consequently, it can be inferred that the social demands puts strong pressure on the teachers and seem to turn their attention to other functions (supportive, sanitary, of social assistance) away from their professional performance and which do not constitute the essence of the social function they attribute to themselves and which they aim at retaining over time.
\end{abstract}

Key words: TEACHERS' KNOWLEDGE; SOCIAL REPRESENTATIONS; NATURAL SCIENCES

\footnotetext{
${ }^{1}$ Licenciada en Psicopedagogía y Doctorando en Educación. Integrante de proyectos de investigación en la Universidad Nacional de San Juan, Argentina. Dirección electrónica: saguilar@ffha.unsj.edu.ar

${ }^{2}$ Doctora en Educación y Profesora en Física. Profesora Titular la Universidad Nacional de San Juan, Argentina. Investigadora del CONICET. Directora proyectos sobre Enseñanza de las Ciencias Naturales. Argentina. Dirección electrónica: mazzitel@ffha.unsj.edu.ar

${ }^{3}$ Licenciada en Sociología, Magíster en Psicología Social y Doctorando en Educación. Docente en escuelas de Nivel Secundario. Integrante de proyectos de investigación en la Universidad Nacional de San Juan, Argentina. Dirección electrónica: chacomas@hotmail.com

${ }^{4}$ Docteur en Éducation, Doctora en Ciencias de la Educación y Máster en Educación, Relaciones Humanas y Comunicación Social. Investigadora del Consejo Nacional de Investigaciones Científicas y Técnicas (CONICET), Argentina.Dirección electrónica: maparici@satlink.com
}

Artículo recibido: 25 de febrero, 2011

Aprobado: 28 de julio, 2011 


\section{Introducción}

La problemática asociada a la enseñanza y al aprendizaje de las Ciencias Naturales es recientemente explorada desde una perspectiva psicosocial, particularmente desde la Teoría de las representaciones sociales (Graça, Moreira y Caballero, 2004; Lacolla, 2005; Martínez Filomeno, 2003; Mazzitelli, 2007), siendo este un abordaje relevante y pertinente para el análisis de estos procesos que son, a la vez, cognitivos y sociales.

Con nuestro equipo de investigación venimos trabajando en esta dirección. En diversos estudios hemos abordado la problemática de las representaciones sociales asociadas a la enseñanza y al aprendizaje de las Ciencias Naturales desde diferentes ángulos.

En primer lugar, identificamos las representaciones sociales de los docentes y estudiantes sobre las Ciencias Naturales (Mazzitelli y Aparicio, 2007) y, luego, avanzamos en la búsqueda de las representaciones sociales de los docentes de Ciencias Naturales en relación con la propia tarea (Mazzitelli, Aguilar, Olivera y Guirado, 2009), el lugar de la Didáctica en las representaciones sociales de los docentes (Mazzitelli, Aguilar, Guirado y Olivera, 2009), las representaciones sociales de docentes y alumnos sobre la escuela (Chacoma, Mazzitelli y Aparicio, 2009), entre otros estudios.

En este trabajo presentamos un análisis comparativo de algunas de nuestras investigaciones. Buscamos identificar aquellos rasgos que forman parte de la estructura de las representaciones sociales de los docentes de Ciencias Naturales y, al mismo tiempo, constituyen elementos comunes que atraviesan estos estudios, presentando una cierta estabilidad independientemente de la temática abordada. Estos elementos nos otorgarán información importante acerca de los significados compartidos por este grupo profesional.

\section{Marco teórico}

\subsection{Las Representaciones Sociales: el saber de sentido común}

En la búsqueda de explicar las relaciones entre pensamiento y cultura, Serge Moscovici (1979) propone un nuevo concepto: las representaciones sociales, que a diferencia de las representaciones colectivas, a las que se refiere Emile Durkheim a finales del siglo XIX, poseen un carácter dinámico, no estático, ya que los sujetos construyen significados y teorías sobre la realidad en una relación dialéctica entre lo individual y lo social. Es decir, las representaciones 
sociales poseen un componente cognitivo y un componente social. Denisse Jodelet (1986) nos ayuda a comprender mejor este concepto señalando

El concepto de representación social designa una forma de conocimiento específico, el saber de sentido común, cuyos contenidos manifiestan la operación de procesos generativos y funcionales socialmente caracterizados. En sentido más amplio, designa una forma de pensamiento social. (...) Las representaciones sociales constituyen modalidades de pensamiento práctico orientados hacia la comunicación, la comprensión y el dominio del entorno social, material e ideal. En tanto que tales, presentan características específicas a nivel de organización de los contenidos, las operaciones mentales y la lógica. (...) La caracterización social de los contenidos o de los procesos de representación ha de referirse a las condiciones y a los contextos en los que surgen las representaciones, a las comunicaciones mediante las que circulan y a las funciones a las que sirven dentro de la interacción con el mundo y los demás (pp. 474-475).

El abordaje de las representaciones sociales nos permitiría acercarnos al modo en que los sujetos interpretan y construyen su conocimiento sobre la realidad y a de que manera esto impacta en sus comportamientos y actitudes frente a los problemas de la vida cotidiana. La representación es, de este modo, una 'organización significante' y una 'guía para la acción' que opera como un sistema de representación, otorgando sentido a las prácticas (Abric, 2001).

Abric (2001) afirma que "la identificación de la 'visión del mundo' que los individuos o grupos llevan en sí y utilizan para actuar (...) es reconocida como indispensable para entender la dinámica de las interacciones sociales y aclarar los determinantes de las prácticas sociales" (p. 11). El autor considera que las representaciones sociales están influenciadas al mismo tiempo por el contexto, tanto discursivo como social. De este modo, las significaciones de las representaciones sociales están mediadas, a la vez, por las características de las condiciones de producción del discurso que nos permite acceder a la representación, por el contexto ideológico y por el lugar del individuo en la sociedad.

Las representaciones sociales, entonces, constituyen construcciones simbólicas que se consolidan a través de diferentes procesos comunicacionales (conversaciones, medios masivos) y se materializan en las prácticas sociales. Mediante la atribución de un sentido particular, las representaciones adquieren independencia del objeto y se arraigan en las interacciones sociales (Petracci y Kornblit, 2007). 
Entre los componentes de las representaciones sociales se encuentran: la información, que es el conjunto de conocimientos de un grupo social en relación con un acontecimiento o fenómeno; el campo o estructura de la representación, que muestra la organización jerárquica del contenido de la representación y la actitud, que se refiere a la orientación positiva o negativa en relación con el objeto de la representación (Mora, 2002; López Alonso y Stefani, 2005, entre otros).

Así, las representaciones sociales articulan la información sobre el objeto de la representación y las actitudes del sujeto y del grupo hacia el objeto.

\subsubsection{La estructura de las Representaciones Sociales}

Como explicamos, las representaciones sociales constituyen un todo estructurado y organizado, compuesto por un conjunto de informaciones, creencias, opiniones y actitudes con relación a un objeto. Abric (2001) afirma que es necesario identificar el contenido y la estructura de estos elementos, porque, además de ser jerarquizados, están organizados alrededor de un núcleo central conformado por algunos elementos que otorgan una significación particular a la representación.

Las creencias, opiniones y actitudes que se identifican en el núcleo central se vinculan, generalmente, a la memoria e historia grupal $y$, por lo tanto, ofrecen una gran resistencia a las transformaciones del contexto social. Esta estabilidad de los elementos garantiza la permanencia y el carácter innegociable de la representación. El núcleo central cumple con dos funciones, por un lado, otorgar el significado a la representación y, por otro, organizar el resto de los elementos (Petracci y Kornblit, 2007).

Por su parte, el sistema periférico tiende a preservar al núcleo de posibles transformaciones, es flexible y variable debido a que es más sensible al contexto inmediato (Petracci y Kornblit, 2007). Entre las funciones del sistema periférico, Abric (2001) menciona:

- Función de concreción: resulta del anclaje de la representación en la realidad.

- Función de regulación: al ser estos elementos más flexibles en comparación con los del núcleo, permiten la adaptación de la representación a las evoluciones del contexto.

- Función de defensa: el sistema periférico funciona como el sistema de protección de la representación, es donde se operará una transformación o donde las contradicciones podrán aparecer y ser sostenidas. 
Finalmente, en la zona de elementos de contraste, encontramos componentes que podrían considerarse como la estructura nuclear de un grupo minoritario (Graca, Morerira y Caballero, 2004).

El abordaje de las representaciones sociales, desde este enfoque, permite diferenciar los elementos más significativos y estables de aquellos más sensibles a los cambios, que presenten características particulares en función de contextos determinados y/o que sirvan de sostén al núcleo central.

\subsubsection{Las funciones de las Representaciones Sociales}

Las representaciones sociales poseen ciertas funciones entre los miembros de la sociedad, relacionadas con las prácticas y las interacciones sociales. Estas funciones muestran cómo las representaciones sociales posibilitan la comprensión del origen de los comportamientos y las prácticas sociales y, al mismo tiempo, se encuentran influidas por éstas. Abric (2001) describe cuatro funciones de las representaciones sociales, estas son:

- Función de saber: describen y explican la realidad y permiten comunicarla, por ello, constituyen un marco que favorece los intercambios y la comunicación social.

- Función identitaria: posibilitan la construcción de una imagen de sí en el grupo, y del grupo en relación a los otros, y un conjunto de valoraciones positivas al respecto.

- Función de orientación: definen la situación y las relaciones a restablecer, producen una anticipación que actúa como filtro de la interpretación de los acontecimientos, y prescribe las prácticas en contextos determinados.

- Función justificadora: operan también luego de la acción con el fin de perpetuar la posición del grupo.

Según el autor mencionado, en general, se encuentran escasas investigaciones que intenten profundizar acerca de la función identitaria de las representaciones sociales. En el caso de los docentes, éstos presentan rasgos característicos en el ejercicio de su profesión que muestran una cierta estabilidad a lo largo del tiempo. La identidad del docente se vincula con un conjunto de representaciones profesionales que le permite reconocerse a sí mismo y, al mismo tiempo, identificarse o diferenciarse de otros grupos profesionales. 


\subsection{Los saberes del docente}

El conocimiento profesional del profesor de Ciencias Naturales está conformado por diferentes componentes: un componente relacionado con el conocimiento de sí mismo, vinculado al reconocimiento de las propias creencias, actitudes y valores referidos a la tarea; un componente académico, que incluye conocimientos científicos, psicopedagógicos y didácticos adquiridos en las distintas instancias de formación y un componente dinámico, relacionado con la capacidad de reflexionar sobre su práctica en contextos escolares concretos. Entre estos componentes existen tensiones al tiempo que interactúan modificándose unos a otros (Mellado Jiménez, Blanco Nieto y Ruiz Macías, 1999).

En relación con el conocimiento de sí mismo, podemos hablar de la identidad profesional. Ésta se construye a lo largo del tiempo desde el inicio de la carrera y durante el ejercicio de la profesión, donde intervienen "diversos procesos de socialización entendidos como procesos biográficos y relacionales, vinculados a un contexto (socio-histórico y profesional)" (Vaillant, 2007 , p. 4). En su desarrollo intervienen procesos de identificación y diferenciación con otras profesiones y constituye un cúmulo de representaciones individuales, producto de la historia personal, y representaciones sociales que otorgan sentido a la tarea en determinados contextos.

Braslavsky y Birgin (1992) explican que los profesores "ponen en acto una epistemología subyacente que le es propia, y que es a la vez producto de una biografía escolar colectiva de la cual participa y de su historia personal" (p. 87). Las autoras, diferencian tres dimensiones de esta epistemología: Los tipos de conocimiento del docente, sus fuentes y sus modos de construcción. Entre los tipos de conocimiento que poseen los docentes encontramos:

- Un saber sustantivo: comprende el dominio de una disciplina, es decir, es el campo de su formación inicial y supone el conocimiento de la estructura epistemológica de ese campo del saber, los ejes conceptuales, los modos de producción y divulgación, etc.

- Un saber pedagógico: supone el encuentro entre los significados subjetivos de los docentes y el conocimiento científico, los modos de apropiación y transposición didáctica del conocimiento, la comprensión del sujeto que aprende, la dinámica áulica y el conocimiento del entorno socio cultural.

- Un saber institucional: las instituciones proveen un marco regulatorio de las actividades que se desarrollan en su seno. En su práctica escolar, los docentes se apropian de los 
saberes relacionados con la historia de la escuela, el contexto social, económico, político, cultural y comunitario que caracterizan esas instituciones y las diferencian de otras.

Algunos de estos tipos de saberes provienen de su formación inicial y continua, otros de la práctica misma y de los intercambios que acontecen en el seno de las instituciones educativas. "Estos saberes 'en acto' se nutren de las ciencias, del sentido común y sobre todo, de la experiencia acumulada en una práctica laboral específica" (Braslavsky y Birgin, 1992, p.90) propia del grupo profesional.

\subsubsection{El saber disciplinar del docente}

Si bien, la formación de profesores ha experimentado una evolución en el campo de la enseñanza de las Ciencias referida a los planteamientos teóricos y a las estrategias de intervención en el aula, es necesaria una formación continua que también incluya contenidos disciplinares, aspecto fundamental en una sociedad cambiante, en la que los conocimientos avanzan y se renuevan constantemente (Mellado, 2000).

Desde una perspectiva amplia, la enseñanza de las Ciencias presenta una encrucijada cognitiva, donde confluyen diversos conocimientos: académico, cotidiano; de las Ciencias, del alumno y del profesor (Marín Martínez, 2003). El contenido que llega al alumno de mano del docente dependerá del grado de comprensión de éste, de sus creencias sobre la enseñanza, el aprendizaje, la construcción del conocimiento de las Ciencias y el modo de enseñarlas.

Muchas veces el profesor, conscientemente o no, transmite a los alumnos conocimientos y una imagen de Ciencia en concordancia con la presentación de los contenidos (Saura Llamas y De Pro Bueno, 2000). En otros casos, el saber científico es reinterpretado a la luz de las concepciones propias de los docentes que, si fueran erradas, podrían involuntariamente contribuir al afianzamiento de las dificultades de los estudiantes. Las formas de razonamiento de los estudiantes son compartidas, con frecuencia, por los docentes, los redactores de libros de textos y los especialistas, produciendo una permanente retroalimentación de sus representaciones (Welti, 2002).

Así, "los docentes pueden favorecer la reelaboración conceptual acercando a los alumnos al conocimiento científico, fortalecer las ideas previas incorrectas $u$ originar nuevas ideas erróneas. Todo dependerá del modo en que promuevan el acercamiento a los conceptos y del 
conocimiento que posean sobre lo que enseñan" (Núñez, Pereira, Maturano y Mazzitelli, 2007, p.1). Por lo tanto, es necesario reflexionar y profundizar sobre el conocimiento del contenido disciplinar del profesorado de Ciencias Naturales, sobre las "ideas" de los docentes en relación con algunos contenidos conceptuales.

\subsubsection{El saber pedagógico}

El saber pedagógico del docente implica un cuestionamiento sobre ¿qué enseña?, ¿cómo lo enseña?, ¿qué se debe enseñar?, ¿qué debe ser y hacer la escuela? Es necesario responder estas preguntas, impregnadas de valores y comprometidas con la ética, no con una intención prescriptiva sino reflexiva (Camilloni, 2007).

Al respecto, Litwin (1998) entiende a la Didáctica como "teoría acerca de las prácticas de la enseñanza significadas en los contextos socio históricos en que se inscriben" (p. 94). Estas prácticas se fundan sobre concepciones "ideológicas" del docente, lo que le permite estructurar el campo y realizar recortes disciplinarios, producto de su historia profesional, ideas y limitaciones.

Para Álvarez Méndez (2001) la Didáctica no puede reducirse a la aplicación de conocimientos de otras disciplinas, sino que implica la construcción de una teoría relativa al quehacer práctico del docente. El autor presenta una visión globalizadora donde en la acción didáctica se consideran tanto el proceso como todos los elementos que intervienen en él y los sujetos (docente y alumno) interactúan.

En este sentido, las representaciones de los docentes acerca de la docencia constituyen un núcleo de significaciones que influyen en las prácticas y en los procesos de interacción en el aula (Kaplan, 2003).

\subsubsection{El saber institucional: La escuela como contexto}

La escuela, según Ana María Brígido (2006), es una parte del complejo sistema educativo, pues la misma responde al conjunto de normas y disposiciones que emergen del propio sistema. Esto implica ubicarla en un contexto social, económico y político que condiciona su ser.

Desde la perspectiva funcionalista, según Brígido (2006: p. 161), “(..) la escuela constituye una unidad integrada por individuos que comparten, en líneas generales, ideas, 
valores, pautas de conducta, etc., y que persiguen, también en líneas generales, objetivos comunes. Los miembros aprenden todo esto a medida que participan en la vida cotidiana de la institución, es decir, mientras son socializados por ella (...)".

En este estudio buscamos explicitar las representaciones sociales de los docentes y a partir de allí reflexionar sobre el modo en que esto incide en sus actitudes y en sus acciones frente a los problemas presentes en el aula de Ciencias.

\section{Metodología}

En el presente trabajo compararemos tres estudios realizados con docentes y con la finalidad de observar las estructuras de las representaciones sociales sobre diferentes temáticas vinculadas a sus saberes, e identificar los elementos comunes en esas estructuras, que se presentan independientemente de la temática planteada.

Para su análisis los hemos organizado considerando la clasificación de saberes del docente de Braslavsky y Birgin (1992). El primer estudio vinculado al saber sustantivo, se refiere a la identificación y análisis de la estructura de las representaciones sociales de docentes sobre las Ciencias Naturales, luego, en relación con el saber pedagógico, la estructura de las representaciones sociales de docentes sobre la Docencia, y finalmente, la estructura de las representaciones sociales de docentes sobre la Escuela, en virtud de su saber institucional.

Hemos realizado un muestreo no probabilístico estratégico o de conveniencia que presenta algunas ventajas, ya que no se precisa de un listado de la población para seleccionar la muestra. Otros aspectos considerados, a fin de lograr una muestra representativa de la población, fueron (Valles, 2000; Mazzitelli, 2007):

- La selección de contextos relevantes al problema de la investigación (niveles educativos en los que trabajan, antigüedad docente, disciplinas de formación).

- La saturación o redundancia, lo que permite no fijar a priori el número de personas que ingresarán a la muestra, sino que este queda determinado una vez que la información comienza a ser redundante y que no aporta ningún nuevo punto de vista analítico.

En el conjunto de investigaciones que analizamos en este artículo, trabajamos con técnicas de evocación y jerarquización de palabras con el objetivo de identificar la estructura nuclear y periférica de las representaciones sociales de docentes. En las distintas estructuras que encontramos, observaremos aquellos rasgos que se presentan en común. 
A continuación, describiremos las formas específicas de abordaje en cada caso.

\subsection{Tratamiento específico de los datos}

3.1.1. La estructura de las representaciones sociales de docentes sobre las Ciencias Naturales

Trabajamos con una muestra de docentes que se estratificó teniendo en cuenta la especialidad de formación (Física, otras Ciencias Naturales y otras disciplinas).

Aplicamos un test de evocación jerarquizada tomando como referencia el propuesto por Graca, Moreira y Caballero (2004). Esta es una técnica cualitativa que permite, a través de procesos de asociación libre y jerarquización, acceder a la estructura de las representaciones sociales.

Al implementarla le solicitamos a los docentes que enumeren la mayor cantidad posible de palabras, con un máximo de 32, que relacionen con un término inductor, en este caso las Ciencias Naturales. Luego, deben realizar selecciones sucesivas para, finalmente, presentar por orden de importancia las palabras que consideran más relacionadas, con un máximo de 8.

Las palabras que resultaron del proceso de selección y jerarquización fueron agrupadas en categorías y, posteriormente, las categorías las agrupamos en cuatro dimensiones. De este análisis llegamos a la identificación de la estructura de las representaciones sociales.

3.1.2. La estructura de las representaciones sociales de docentes de Ciencias sobre la Docencia

Para este estudio la muestra fue estratificada teniendo en cuenta el nivel educativo en el que concentran su mayor carga horaria (nivel secundario, nivel universitario), cabe destacar que los docentes universitarios de la muestra son docentes de profesorados (formadores de formadores) y la antigüedad en el ejercicio de la docencia (expertos y novatos)

Trabajamos con una técnica de evocación y jerarquización, que permite acceder a la estructura de las representaciones sociales de un determinado grupo (Abric, 2001; Mazzitelli, 2007; Petracci y Kornblit, 2007). Para esto le solicitamos a los docentes que mencionaran, en orden de importancia, cinco palabras que asociaran a la 'Docencia'. A partir de las palabras relevadas a través de esta técnica elaboramos categorías que permitieran agruparlas y ordenarlas. 
Una vez elaboradas las categorías, procedimos a agruparlas en dimensiones que sirvieron al análisis de las estructuras de las representaciones sociales de los docentes sobre 'Docencia'.

\subsubsection{La estructura de las representaciones sociales de docentes sobre la Escuela}

Consideramos que las representaciones sociales de los docentes, acerca de la Escuela, varían según el contexto socio-cultural donde se inserta la escuela en la que se desempeñan y, además, estas representaciones constituyen uno de los factores obstaculizadores del aprendizaje. Por esta razón, hemos trabajado con docentes de una escuela urbana y de otra urbana con vulnerabilidad social ${ }^{5}$.

Al igual que en el estudio realizado para la identificación de las representaciones sociales sobre las Ciencias Naturales, implementamos un test de evocación jerarquizada (Graca, Moreira y Caballero, 2004 y Mazzitelli, 2007), que, como ya dijimos, es una técnica que permite acceder a la estructura de la representaciones sociales de un determinado grupo (Abric, 2001).

En este caso, el término inductor fue la Escuela. Las palabras que resultaron del proceso de selección y jerarquización fueron agrupadas en categorías y subcategorías. A partir de ellas se construyeron las estructuras de las representaciones sociales de los docentes sobre la 'Escuela'.

\subsection{Tratamiento general de los datos}

El primer paso en el procesamiento de los datos, en todos los casos, fue ordenar y agrupar las palabras en categorías. Luego, evaluamos la frecuencia de aparición y el nivel de importancia asignado para poder llegar a identificar la estructura de la representación social.

El criterio utilizado para decidir cuándo la frecuencia de aparición de cada categoría se consideraría alta o baja, fue tener en cuenta las frecuencias de todas las categorías, para la

5 En Argentina estas escuelas reciben la denominación de escuelas urbano-marginales. Tras diversos cambios en la sociedad y en las escuelas, en los últimos tiempos, apareció en Argentina la clasificación de escuelas urbanas y urbano-marginales (Tenti Fanfani, 2007). Por escuelas urbanas se entiende aquellas escuelas que se encuentran ubicadas en zonas urbanas y la población de alumnos que concurre pertenece a sectores sociales con ingresos económicos medios y medio-altos, contando en sus viviendas con servicios de agua potable, planificación edilicia, acceso a vías rápidas de comunicación de todo tipo sea tecnológico como de transporte, etc. Por escuelas urbanomarginales se entiende a aquellos establecimientos educativos ubicados en zonas urbanas y a los que asiste una población de alumnos procedente de sectores sociales carenciados o vulnerables. 
muestra general y para cada submuestra de docentes por separado (nivel secundario/nivel universitario; expertos/novatos y Física/otras Ciencias Naturales/otras disciplinas).

En cada caso, calculamos el promedio (en adelante lo representaremos por $p$ ) entre la mayor y la menor frecuencia de las categorías. Luego, consideramos que si $n$ (frecuencia de aparición de la categoría para la muestra general o para cada submuestra, según el análisis que fuéramos a realizar) es mayor o igual a $p$, la frecuencia es alta y si $n$ es menor que $p$, la frecuencia es baja (Graca, Moreira y Caballero, 2004).

Mientras tanto, en relación con la importancia asignada a cada categoría, consideramos los promedios para cada una de ellas, obtenidos de los valores de importancia asignados a cada una de las palabras que ingresaron a las respectivas categorías (teniendo en cuenta el orden en que fueron jerarquizadas por los docentes). Es decir, para cada categoría, de forma independiente, promediamos la importancia asignada a las palabras que ingresaron a esa categoría y la comparamos con el valor medio del promedio de palabras mencionadas por los docentes.

A posteriori de la elaboración de las categorías, teniendo en cuenta la frecuencia de aparición de las palabras para cada una de las mismas y el orden de importancia asignado, procedimos al agrupamiento de éstas en cuatro zonas:

- Zona I: Frecuencia Alta -Importancia Grande (Núcleo).

- Zona II: Frecuencia Alta -Importancia Pequeña (Primera Periferia).

- Zona III: Frecuencia Baja -Importancia Grande (Segunda Periferia).

- Zona IV: Frecuencia Baja-Importancia Pequeña (Elementos de Contraste).

En algunos de los estudios realizados, como ya adelantamos, además de agrupar las palabras en categorías, elaboramos dimensiones a partir de las cuales pudimos asociar las categorías. Esto permite realizar un análisis de las estructuras rescatando los aspectos más generales, lo que facilita, por un lado, la comparación de las estructuras de los distintos subgrupos en los que se estratificaron las muestras de docentes con las que trabajamos reconociendo diferencias y semejanzas-y, por otro lado, llegar a identificar mejor la significación de las representaciones sociales, infiriendo algunas posibles derivaciones. 


\subsection{Elaboración de categorías y dimensiones}

\subsubsection{Sobre las Ciencias Naturales}

A partir del término inductor 'Ciencias Naturales', con las palabras mencionadas por los docentes elaboramos las categorías que se observan en el siguiente cuadro:

\section{Cuadro No. 1}

\section{Categorías construidas en torno al término 'Ciencias Naturales'}

\begin{tabular}{|c|c|}
\hline Categoría & Definición \\
\hline Conceptos generales I & Conceptos no exclusivos de las Ciencias. \\
\hline Conceptos generales II & Vocablos vinculados al dominio de las Ciencias Naturales. \\
\hline Conceptos específicos & Palabras relacionadas con una parte de las Ciencias Naturales. \\
\hline Objetos de estudio & Elementos "concretos" que "estudian" las Ciencias Naturales. \\
\hline Procedimientos & $\begin{array}{l}\text { Palabras que se refieran a los procedimientos propios de las Ciencias, en general, y } \\
\text { de las Ciencias Naturales en particular. }\end{array}$ \\
\hline Herramientas & "Accesorios" en los que buscan ayuda las Ciencias Naturales. \\
\hline Estructura teórica & Conceptos de la estructura teórica de las Ciencias. \\
\hline $\begin{array}{l}\text { H. Partes } \\
\text { (disciplinas) }\end{array}$ & Disciplinas específicas incluidas en las Ciencias Naturales. \\
\hline Enseñanza y aprendizaje & $\begin{array}{l}\text { Palabras relacionadas con los procesos de enseñanza y de aprendizaje de las } \\
\text { Ciencias Naturales. }\end{array}$ \\
\hline Nombres de científicos. & Nombres propios de personas asociadas a las Ciencias. \\
\hline Actitudes positivas & $\begin{array}{l}\text { Palabras que implican una disposición favorable hacia las Ciencias Naturales y/o hacia } \\
\text { su enseñanza y su aprendizaje. }\end{array}$ \\
\hline Actitudes negativas & $\begin{array}{l}\text { Palabras que implican una disposición desfavorable hacia las Ciencias Naturales y/o } \\
\text { hacia su enseñanza y su aprendizaje. }\end{array}$ \\
\hline
\end{tabular}

Fuente: Elaboración de los autores de este artículo

Luego, agrupamos estas categorías en cuatro dimensiones (Graca, Moreira y Caballero, 2004):

- Epistemológica: cuestiones que se relacionan con el aspecto formal de las Ciencias y la construcción del conocimiento científico (Categorías b., c., d., e., f., g. y h.).

- Pedagógica: aspectos vinculados con la enseñanza y el aprendizaje de las Ciencias Naturales, desde las cuestiones formales de estos procesos hasta las relaciones que 
se establecen entre los actores entre sí y entre estos y el conocimiento escolar (Categoría i.).

- Afectiva: relacionado con la valoración o desvalorización del conocimiento de las Ciencias Naturales y de su enseñanza y su aprendizaje (Categorías k. y I.).

- Socio-cultural: aspectos que tienen que ver con la interacción y la comunicación entre las personas de un grupo social y con la actividad y el bagaje cultural de una sociedad (Categorías a. y j.).

\subsubsection{Sobre la Docencia}

A partir de las palabras relevadas a través de esta técnica elaboramos categorías que permitieran agruparlas y ordenarlas. Las categorías obtenidas se muestran en el cuadro a continuación:

Cuadro No. 2

Categorías construidas en torno al término 'Docencia'

\begin{tabular}{|l|l|}
\hline \multicolumn{1}{|c|}{ Categorías } & \\
\hline 1.Interacción & Relacionadas con la interacción entre los distintos actores asociados al quehacer docente. \\
\hline 2.Valores & Palabras que señalan valores positivos. \\
\hline 3.Actitudes & Muestran actitudes hacia la docencia, es decir, de los docentes hacia su quehacer. \\
\hline 4.Sujetos & Aluden a los actores del quehacer educativo. \\
\hline 5.Conocimiento & Palabras referidas al quehacer docente en relación con el conocimiento. \\
\hline 6.Formación & Asociadas a la formación y perfeccionamiento docente. \\
\hline 7.Enseñanza & $\begin{array}{l}\text { Palabras referidas al proceso de enseñanza, desde una perspectiva más amplia que incluye } \\
\text { distintas posturas al respecto. }\end{array}$ \\
\hline 8.Aprendizaje & Palabras vinculadas al proceso de aprendizaje, también desde una perspectiva general. \\
\hline 9.Fines & Muestran los objetivos o fines a los que tiende la docencia. \\
\hline 10.Didáctica & Palabras que aluden a elementos o actividades propios de la práctica docente. \\
\hline 11.Significados & Señalan significaciones estereotipadas sobre la docencia. \\
\hline
\end{tabular}

Fuente: Elaboración de los autores de este artículo 
Una vez elaboradas las categorías, procedimos a agruparlas en dimensiones (Graca, Moreira y Caballero, 2004; Mazzitelli, 2007). Las dimensiones elaboradas y los criterios considerados son:

- Dimensión Identitaria. Las categorías incluidas son: significados, valores, actitudes. Agrupa a aquellos aspectos vinculados con la identificación con el rol profesional.

- Dimensión Educativa. Incluye las categorías enseñanza, aprendizaje, formación. Concierne al hecho educativo en sí mismo, que comprende los procesos de enseñanza y aprendizaje.

- Dimensión Curricular. Se refiere a los elementos que comprende un curriculum: para qué, qué y cómo se enseña. Las categorías incluidas son fines, conocimiento, didáctica.

- Dimensión Vincular. Tiene que ver con los sujetos que participan y sus interacciones. Agrupamos las categorías interacción y sujetos.

\subsubsection{Sobre la Escuela}

A partir de la técnica de evocación de palabras explicada, elaboramos las siguientes categorías y subcategorías para agruparlas. Las mismas se presentan en el Cuadro No. 3.

Cuadro No. 3

Categorías construidas en torno al término 'Escuela'

\begin{tabular}{|c|c|}
\hline Categorías y Subcategorías & Definición \\
\hline 1-Comunidad Educativa & Hacen referencia a los actores propios de la vida escolar. \\
\hline 2-Función de la Escuela & Hacen referencia a las diversas demandas a las que la Escuela debe responder. \\
\hline 2.1- Educativa & $\begin{array}{l}\text { Asociadas a los procesos de enseñanza y de aprendizaje y a la Escuela como } \\
\text { Institución formadora. }\end{array}$ \\
\hline 2.2- Socializadora & Hacen referencia al aprendizaje de pautas, normas, valores sociales. \\
\hline 2.3- Normativa & $\begin{array}{l}\text { Vinculadas a las normas y reglas de conducta necesarias para la convivencia en la } \\
\text { escuela. }\end{array}$ \\
\hline 3-Caracterización de la Escuela & Referidas a las características que se le atribuyen a la Escuela. \\
\hline 3.1- Positiva & Aspectos que favorecen las actividades de enseñanza y de aprendizaje. \\
\hline 3.2- Negativa & Aspectos que dificultan las actividades de enseñanza y de aprendizaje. \\
\hline $\begin{array}{l}\text { 4-Elementos que asocian a la } \\
\text { Actividad Educativa }\end{array}$ & $\begin{array}{l}\text { Referidas a los elementos materiales que contribuyen con los procesos de } \\
\text { enseñanza y de aprendizaje. }\end{array}$ \\
\hline $\begin{array}{l}\text { 5-Actividades del Docente en la } \\
\text { Escuela }\end{array}$ & Hacen referencia a las actividades que el docente lleva a cabo dentro de la Escuela. \\
\hline 6-Actividades que los Docentes & Asociadas a actividades que los docentes vinculan con la Escuela aunque no son \\
\hline
\end{tabular}




\begin{tabular}{|c|l|}
\hline asocian a la Escuela & inherentes a ella. \\
\hline 6.1- Positivas & $\begin{array}{l}\text { Actividades o acciones que el docente asocia a su lugar de trabajo, la Escuela con } \\
\text { una valoración positiva. }\end{array}$ \\
\hline 6.2-Negativas & $\begin{array}{l}\text { Actividades o acciones que el docente asocia a su lugar de trabajo, la Escuela con } \\
\text { una valoración negativa. }\end{array}$ \\
\hline 7-Contexto de la Escuela: & Se refieren al contexto extraescolar en que se sitúan los actores de la Escuela. \\
\hline $\begin{array}{l}\text { 8-Valores Predominantes en la } \\
\text { Escuela }\end{array}$ & $\begin{array}{l}\text { Expresan valores, es decir, principios generales que orientan las acciones sociales de } \\
\text { las personas, los diferentes grupos y sociedades. }\end{array}$ \\
\hline 8.1- Positivos & $\begin{array}{l}\text { Expresan valores positivos que orientan las acciones sociales de las personas en la } \\
\text { Escuela. }\end{array}$ \\
\hline 8.2- Negativos & $\begin{array}{l}\text { Relacionadas a valores negativos que orientan las acciones de los diferentes grupos } \\
\text { en la Escuela. }\end{array}$ \\
\hline
\end{tabular}

Fuente: Elaboración de los autores de este artículo

Comparando las categorías elaboradas a partir de las palabras enumeradas por los docentes para término inductor, observamos que el aspecto que aparece como elemento común es el referido a la enseñanza y el aprendizaje.

Seguidamente, presentamos y analizamos las estructuras de las representaciones sociales identificadas, considerando la presencia de las categorías que aparecen como elementos comunes.

\section{Resultados y su análisis}

\subsection{Estructuras de las representaciones sociales sobre las Ciencias Naturales}

Presentamos las estructuras, de las representaciones sociales identificadas, diferenciadas según la disciplina de formación de los docentes de la muestra.

Tabla No. 1

Estructura de las representaciones sociales de docentes de Física

\begin{tabular}{|c|c|c|}
\hline & IMPORTANCIA GRANDE & IMPORTANCIA PEQUEÑA \\
\hline $\begin{array}{c}\text { FRECUENCIA } \\
\text { ALTA }\end{array}$ & $\begin{array}{c}\text { ZONA I (Núcleo) } \\
\text { Objetos de estudio } \\
\text { Conceptos generales II }\end{array}$ & $\begin{array}{c}\text { ZONA II (Primera Periferia) } \\
\text { Procedimientos } \\
\text { Partes }\end{array}$ \\
Conceptos específicos \\
\hline $\begin{array}{c}\text { FRECUENCIA } \\
\text { BAJA }\end{array}$ & $\begin{array}{c}\text { ZONA IV } \\
\text { (Elementos de contraste) } \\
\text { Conceptos generales I } \\
\text { Actitudes positivas }\end{array}$ & $\begin{array}{c}\text { ZONA III } \\
\text { Estructura teórica } \\
\text { Herramientas }\end{array}$ \\
\hline
\end{tabular}

Fuente: Elaboración de los autores de este artículo 
Tabla No. 2

Estructura de las representaciones sociales de docentes de otras Ciencias Naturales

\begin{tabular}{|c|c|c|}
\hline & IMPORTANCIA GRANDE & IMPORTANCIA PEQUEÑA \\
\hline FRECUENCIA & ZONA I (Núcleo) & ZONA II (Primera Periferia) \\
ALTA & Objetos de estudio & Estructura teórica \\
& Partes & Procedimientos \\
& Conceptos generales II & Conceptos específicos \\
\hline FRECUENCIA & ZONA IV(Elementos de contraste) & ZONA III(Segunda Periferia) \\
BAJA & Conceptos generales I & \\
& Actitudes positivas & \\
\hline
\end{tabular}

Fuente: Elaboración de los autores de este artículo

Tabla No. 3

Estructura de las representaciones sociales de docentes de otras disciplinas

\begin{tabular}{|c|c|c|}
\hline & IMPORTANCIA GRANDE & IMPORTANCIA PEQUEÑA \\
\hline $\begin{array}{c}\text { FRECUENCIA } \\
\text { ALTA }\end{array}$ & $\begin{array}{c}\text { ZONA I (Núcleo) } \\
\text { Objetos de estudio } \\
\text { Partes }\end{array}$ & $\begin{array}{c}\text { ZONA II (Primera Periferia) } \\
\text { Procedimientos } \\
\text { Conceptos específicos }\end{array}$ \\
\hline $\begin{array}{c}\text { FRECUENCIA } \\
\text { BAJA }\end{array}$ & $\begin{array}{c}\text { Conceptos Generales I } \\
\text { Enseñanza y Aprendizaje } \\
\text { Herramientas }\end{array}$ & EONtructura teórica \\
& Conceptos generales II & Actitudes positivas \\
& Nombres de científicos
\end{tabular}

Fuente: Elaboración de los autores de este artículo

Al analizar estas estructuras observamos que aunque el término inductor estaba dirigido directamente al saber sustantivo del docente, es decir, al saber disciplinar, aparecieron palabras vinculadas con su enseñanza y aprendizaje, que fueron contenidas en la dimensión Pedagógica.

Sin embargo, no aparecen en el Núcleo, sino que se muestran en la Segunda periferia, más susceptible a los cambios, en el grupo de docentes de Física y en la Zona de los elementos de contraste, para los docentes de otras disciplinas, lo que implicaría la presencia de un grupo minoritario que posee una representación social diferente. En cambio, está ausente en la estructura de los docentes de otras Ciencias Naturales. 
Volviendo al Núcleo, en esta zona predominan las cuestiones más formales como las Partes o disciplinas más específicas, los Conceptos Generales II y los Objetos de estudio, categorías vinculadas con la dimensión Epistemológica.

Al diferenciar las estructuras de las representaciones sociales, según la disciplina de formación y el nivel educativo en el que se desempeñan los docentes (no incluimos las tablas por la extensión del trabajo), identificamos que existen diferencias entre las estructuras de los docentes de nivel secundario y de nivel universitario. Cabe destacar que los docentes universitarios, a quienes se les aplicó el test de evocación jerarquizada, son docentes de profesorados (formadores de formadores).

Entre las diferencias que rescatamos en las estructuras de las representaciones sociales encontramos que para los docentes de Física, y de otras disciplinas de nivel universitario, no aparece en ninguna de las zonas la categoría Enseñanza y Aprendizaje.

\subsection{Estructuras de las representaciones sociales sobre la Docencia}

A continuación, presentamos las estructuras identificadas. En un primer momento analizamos la estructura general para todos los docentes $\mathrm{y}$, posteriormente, las estructuras diferenciadas según la antigüedad de los docentes en el ejercicio profesional (novato/experto) y el nivel educativo en el que se desempeñan (universitario/secundario).

\subsubsection{Estructura general de todos los docentes}

Al analizar esta estructura observamos que en el Núcleo aparecen categorías asociadas a las dimensiones Educativa e Identitaria, siendo mayor la presencia de la primera. Al respecto, podemos decir que las representaciones sociales de los docentes acerca de la 'Docencia' aparecen vinculadas principalmente con el enseñar, el aprender y los significados estereotipados asociados al ser docente.

En la primera periferia aparecen categorías que refuerzan el Núcleo en relación con la dimensión Identitaria y recién en la segunda periferia aparecen las categorías que se asocian a las dimensiones Curriculary Vincular. 
Tabla No. 4

Estructura de las representaciones sociales de los docentes sobre la 'Docencia'

\begin{tabular}{|c|c|c|}
\hline & IMPORTANCIA GRANDE & IMPORTANCIA PEQUEÑA \\
\hline $\begin{array}{c}\text { FRECUENCIA } \\
\text { ALTA }\end{array}$ & $\begin{array}{l}\text { ZONA I (Núcleo) } \\
\text { Enseñanza } \\
\text { Aprendizaje } \\
\text { Significados } \\
\end{array}$ & $\begin{array}{c}\text { ZONA II (Primera Periferia) } \\
\text { Valores } \\
\text { Actitudes }\end{array}$ \\
\hline $\begin{array}{c}\text { FRECUENCIA } \\
\text { BAJA }\end{array}$ & $\begin{array}{l}\text { ZONA IV (Elementos de contraste) } \\
\text { Formación }\end{array}$ & $\begin{array}{c}\text { ZONA III (Segunda Periferia) } \\
\text { Didáctica } \\
\text { Fines } \\
\text { Conocimiento } \\
\text { Interacción } \\
\text { Sujetos }\end{array}$ \\
\hline
\end{tabular}

Fuente: Elaboración de los autores de este artículo

Como vemos, en el núcleo de las representaciones sociales encontramos cuestiones que si bien están relacionadas con la práctica educativa aparecen como conceptos más generales (enseñar, proceso de aprendizaje, apostolado, entre otras muchas). Los aspectos curriculares (el qué enseño, cómo enseño, para qué enseño) y vinculares (a quién enseño, quién enseña, quién aprende), que hacen más a la práctica educativa concreta, no están presentes en el Núcleo.

La Formación queda fuera de la estructura de las representaciones sociales "predominante", en la Zona de los elementos de contraste.

\subsubsection{Estructuras de docentes novatos y expertos}

Tabla No. 5

\section{Estructura de las representaciones sociales de los docentes novatos sobre la 'Docencia'}

\begin{tabular}{|c|c|c|}
\hline & IMPORTANCIA GRANDE & IMPORTANCIA PEQUEÑA \\
\hline FRECUENCIA ALTA & $\begin{array}{c}\text { ZONA I (Núcleo) } \\
\text { Enseñanza } \\
\text { Aprendizaje } \\
\text { Significados } \\
\end{array}$ & $\begin{array}{c}\text { ZONA II (Primera Periferia) } \\
\text { Valores } \\
\text { Actitudes }\end{array}$ \\
\hline FRECUENCIA BAJA & ZONA IV (Elementos de contraste) & $\begin{array}{c}\text { ZONA III (Segunda Periferia) } \\
\text { Conocimiento } \\
\text { Formación } \\
\text { Fines } \\
\text { Didáctica } \\
\text { Interacción } \\
\text { Sujetos }\end{array}$ \\
\hline
\end{tabular}

Fuente: Elaboración de los autores de este artículo 
Tabla No. 6

Estructura de las representaciones sociales de los docentes expertos sobre la 'Docencia'

\begin{tabular}{|c|c|c|}
\hline & IMPORTANCIA GRANDE & IMPORTANCIA PEQUEÑA \\
\hline \multirow{2}{*}{ FRECUENCIA ALTA } & $\begin{array}{c}\text { ZONA I (Núcleo) } \\
\text { Enseñanza } \\
\text { Aprendizaje } \\
\text { Significados } \\
\text { Formación }\end{array}$ & Actitudes \\
\hline FRECUENCIA BAJA & ZONA IV (Elementos de contraste) & $\begin{array}{c}\text { ZONA III (Segunda Periferia) } \\
\text { Conocimiento } \\
\text { Fines } \\
\text { Didáctica } \\
\text { Interacción } \\
\text { Sujetos }\end{array}$ \\
\hline
\end{tabular}

Fuente: Elaboración de los autores de este artículo

Al comparar las estructuras según la antigüedad en el ejercicio docente vemos una similitud entre ellas, especialmente en el Núcleo donde aparecen categorías correspondientes a las dimensiones Educativa e Identitaria.

Las diferencias que podemos señalar son que en el Núcleo de la estructura de los docentes expertos se fortalece la dimensión Educativa con la incorporación de la categoría Formación, mientras que esta categoría aparece en la segunda periferia para los docentes novatos. En la primera periferia encontramos que para los docentes expertos sólo aparecen las Actitudes.

\subsubsection{Estructuras de docentes de nivel universitario y de nivel secundario}

Comparando las estructuras según el nivel en el que los docentes concentran mayor carga horaria vemos una diferencia en el Núcleo ya que, si bien para ambos grupos aparecen categorías asociadas a las dimensiones Educativa e Identitaria, en el caso de los docentes de nivel universitario encontramos la categoría Sujetos que corresponde a la dimensión Vincular. Esta categoría para los docentes de nivel secundario aparece fuera de la estructura predominante. 
Tabla No. 7

Estructura de las representaciones sociales de los docentes de nivel universitario sobre la 'Docencia'

\begin{tabular}{|c|c|c|}
\hline & IMPORTANCIA GRANDE & IMPORTANCIA PEQUEÑA \\
\hline FRECUENCIA ALTA & $\begin{array}{c}\text { ZOA I (Núcleo) } \\
\text { Enseñanza } \\
\text { Significados } \\
\text { Sujetos }\end{array}$ & $\begin{array}{c}\text { ZONrendizaje } \\
\text { Actitudes }\end{array}$ \\
\hline FRECUENCIA BAJA & ZONA IV (Elementos de contraste) & $\begin{array}{c}\text { ZONA III (Segunda Periferia) } \\
\text { Conocimiento } \\
\text { Didáctica } \\
\text { Interacción } \\
\text { Valores }\end{array}$ \\
\end{tabular}

Fuente: Elaboración de los autores de este artículo

Tabla No. 8

Estructura de las representaciones sociales de los docentes de nivel secundario sobre la 'Docencia'

\begin{tabular}{|c|c|c|}
\hline & IMPORTANCIA GRANDE & IMPORTANCIA PEQUEÑA \\
\hline FRECUENCIA ALTA & $\begin{array}{l}\text { ZONA I (Núcleo) } \\
\text { Enseñanza } \\
\text { Aprendizaje } \\
\text { Significados }\end{array}$ & $\begin{array}{c}\text { ZONA II (Primera Periferia) } \\
\text { Valores } \\
\text { Actitudes }\end{array}$ \\
\hline FRECUENCIA BAJA & $\begin{array}{c}\text { ZONA IV (Elementos de contraste) } \\
\text { Sujetos } \\
\text { Formación }\end{array}$ & $\begin{array}{c}\text { ZONA III (Segunda Periferia) } \\
\text { Conocimiento } \\
\text { Fines } \\
\text { Didáctica } \\
\text { Interacción }\end{array}$ \\
\hline
\end{tabular}

Fuente: Elaboración de los autores de este artículo

\subsection{Estructuras de las representaciones sociales sobre la Escuela}

En este punto analizamos las estructuras de las representaciones sociales de los docentes de nivel secundario, diferenciadas según el contexto socio-cultural donde se inserta la escuela en la que se desempeñan. 
4.3.1. Estructura de las representaciones sociales sobre la Escuela, de docentes de una escuela urbana

Tabla No. 9

Estructura de las representaciones sociales de docentes de una escuela urbana.

\begin{tabular}{|c|c|c|}
\hline & $\begin{array}{l}\text { IMPORTANCIA } \\
\text { GRANDE }\end{array}$ & IMPORTANCIA PEQUEÑA \\
\hline $\begin{array}{c}\text { FRECUENCIA } \\
\text { ALTA }\end{array}$ & $\begin{array}{l}\text { ZONA I (Núcleo) } \\
\text { Comunidad Educativa } \\
\text { Función de la Escuela } \\
\text { Educativa }\end{array}$ & $\begin{array}{c}\text { ZONA II (Primera Periferia) } \\
\text { Caracterización Positiva de la Escuela } \\
\text { Actividades del docente en la Escuela } \\
\text { Valores Positivos Predominantes en la Escuela }\end{array}$ \\
\hline $\begin{array}{c}\text { FRECUENCIA } \\
\text { BAJA }\end{array}$ & $\begin{array}{c}\text { ZONA IV (Elementos de } \\
\text { contraste) } \\
\text { Función de la Escuela } \\
\text { Socializadora } \\
\text { Valores Negativos } \\
\text { Predominantes en la Escuela }\end{array}$ & $\begin{array}{c}\text { ZONA III (Segunda Periferia) } \\
\text { Función de la Escuela Normativa } \\
\text { Características Negativas de la Escuela } \\
\text { Instrumentos que asocian a la Actividad Educativa } \\
\text { Actividades Positivas que los docentes asocian a la } \\
\text { Escuela } \\
\text { Actividades Negativas que los docentes asocian a la } \\
\text { Escuela } \\
\text { Contexto de la Escuela }\end{array}$ \\
\hline
\end{tabular}

Fuente: Elaboración de los autores de este artículo

En la estructura de las representaciones sociales de los docentes de una escuela urbana, encontramos que su núcleo está conformado por las mismas categorías que las de los docentes de la escuela urbana con vulnerabilidad social, es decir, Comunidad Educativa y Función de la Escuela Educativa.

Por lo tanto, al igual que en el caso anterior, vemos que los docentes consideran que la principal función de la Escuela sigue siendo la educativa y, además, le otorgan una alta significación a la comunidad escolar a la que pertenecen y en la que desempeñan su labor, como también a todos los actores que comparten su tarea.

En el sistema periférico los docentes consideran que la función de la Escuela va más allá de la formación en contenidos y que debe rescatar los valores que hacen a la democracia y la participación, como también seguir siendo una institución que ofrezca posibilidades de desarrollo y progreso. Esto le demanda al docente la apropiación de esa realidad (valores, creencias y símbolos de la situación en la que enseña) en la que debe desempeñar su profesión y en la cual debe "salvaguardar" la función principal de la Escuela, permitiendo integrar a los alumnos al sistema de valores, creencias y normas compartidos por la sociedad en general. 
4.3.2. Estructura de las representaciones sociales sobre la Escuela de docentes de una escuela urbana con vulnerabilidad social

Tabla No. 10

Estructura de las representaciones sociales de docentes de una escuela urbana con vulnerabilidad social

\begin{tabular}{|c|c|c|}
\hline & IMPORTANCIA GRANDE & IMPORTANCIA PEQUEÑA \\
\hline $\begin{array}{l}\text { FRECUENCIA } \\
\text { ALTA }\end{array}$ & $\begin{array}{c}\text { ZONA I (Núcleo) } \\
\text { Comunidad Educativa } \\
\text { Función de la Escuela Educativa }\end{array}$ & $\begin{array}{l}\text { ZONA II (Primera Periferia) } \\
\text { Función de la Escuela Socializadora }\end{array}$ \\
\hline $\begin{array}{c}\text { FRECUENCIA } \\
\text { BAJA }\end{array}$ & $\begin{array}{c}\text { Contexto de la Escuela } \\
\text { Valores Positivos Predominantes en la } \\
\text { Escuela }\end{array}$ & $\begin{array}{c}\text { ZONA III (Segunda Periferia) } \\
\text { Función de la Escuela Normativa } \\
\text { Características Positivas de la Escuela } \\
\text { Características Negativas de la Escuela } \\
\text { Instrumentos que asocian a la Actividad } \\
\text { Educativa } \\
\text { Actividad del docente en la Escuela } \\
\text { Actividades Positivas que los docentes asocian } \\
\text { a la Escuela } \\
\text { Actividades Negativas que los docentes } \\
\text { asocian a la Escuela } \\
\text { Valores Negativos predominantes en la } \\
\text { Escuela }\end{array}$ \\
\hline
\end{tabular}

Fuente: Elaboración de los autores de este artículo

Al considerar esta estructura de las representaciones sociales vemos que en su núcleo aparecen las categorías Comunidad Educativa y Función de la Escuela: Educativa.

Respecto de la primera categoría (Comunidad Educativa), podemos decir que los docentes le otorgan una alta significación a la comunidad escolar a la que pertenecen y en la que desempeñan su labor, como también a todos los actores que comparten su quehacer.

A partir de la segunda categoría, Función de la Escuela: Educativa, podríamos inferir que para los docentes la principal función de la Escuela sigue siendo la educativa, esto permite entender cuál es, para ellos, su "que hacer" en la Escuela, lo que se corresponde con su formación y coincide con el objetivo para el cual la Escuela ha sido históricamente creada, el de educar.

En lo que respecta a las zonas restantes, se hace evidente una relación con la naturaleza del Núcleo, en el cual la cultura y las normas del entorno social condicionan su conformación, ya que los elementos periféricos poseen informaciones seleccionadas e interpretadas, que le permiten al docente realizar juicios con respecto a la Escuela y su entorno. 


\subsection{Comparación y discusión de los resultados parciales}

Hemos identificado que en la estructura de las representaciones sociales de los docentes sobre las Ciencias Naturales, predomina la dimensión Epistemológica, es decir, responde a un esquema formal de estas Ciencias, apareciendo las otras tres dimensiones (Pedagógica, Afectiva y Socio-cultural) como aspectos de poca importancia o ausentes.

La categoría Enseñanza y aprendizaje aparece en los docentes de Física en la segunda periferia, en los elementos de contraste entre los docentes de otras disciplinas, y está ausente en los docentes de Otras Ciencias Naturales. No encontramos en el núcleo aquellos aspectos que favorecerían un mayor interés de los alumnos por el aprendizaje de las Ciencias Naturales, como la conexión con la vida cotidiana y con la cultura, el interés por el conocimiento y por su enseñanza. Sin embargo, podemos afirmar que el saber pedagógico está presente en la estructura acerca del conocimiento disciplinar, a pesar de no haber sido consultados específicamente sobre ello.

Mientras tanto, si comparamos las diversas estructuras de las representaciones sociales analizadas en relación con la Docencia, vemos una gran similitud en el núcleo central, predominando los aspectos asociados a las dimensiones Educativa e Identitaria. Solamente al contrastar las estructuras, según el nivel educativo en el que se desempeñan los profesores, encontramos una diferencia en el núcleo de los docentes de nivel universitario en el que se agrega un elemento (Sujetos) perteneciente a la dimensión Vincular.

Con respecto a los elementos periféricos, observamos que los que aparecen en la primera periferia, reforzando el núcleo, corresponden, en general, a la dimensión Identitaria. En la segunda periferia aparecen, en la mayoría de las estructuras, elementos pertenecientes a las dimensiones Vincular y Curricular. Existe una complementariedad entre los elementos periféricos y los del núcleo lo que muestra que este último es el que le otorga sentido a la representación.

Por último, realizando un análisis general de las estructuras de las representaciones sociales identificadas acerca de la Escuela, podemos decir que los docentes de nuestra muestra consideran que la función principal, independientemente del contexto en el que se encuentre inserta la Escuela, es la Educativa. No obstante, esta función va más allá de la formación en contenidos, y pone de relieve la importancia de los distintos actores de la institución educativa y sus interacciones. 
Las diferencias en el resto de la estructura se pueden asociar a sus funciones de concreción, regulación y defensa, ya que incorpora los elementos dependientes del contexto particular de cada tipo de Escuela, permitiendo así la adaptación de la representación a los cambios del mismo y protegiendo de transformaciones profundas al núcleo central.

A partir de estos resultados podemos afirmar que el saber pedagógico, vinculado con la enseñanza y el aprendizaje de las Ciencias, aparece como elemento común entre estos estudios. Como indicamos, el saber pedagógico implica una serie de significados compartidos por los docentes acerca de su práctica y le otorga un sentido particular a su quehacer, diferenciándolos de otros grupos profesionales.

\section{Conclusiones}

Al realizar un análisis transversal de los núcleos de las estructuras de las representaciones sociales, aparecen elementos que se mantienen más allá de las temáticas planteadas en cada caso particular o las diferentes muestras de docentes. Las categorías que se presentan de modo constante se vinculan directamente en la práctica profesional docente con el hecho de "educar", es decir, formar sujetos escolarizados para el mundo social en el cual se está inserto.

Así, en el primer estudio, si bien los docentes fueron consultados sobre la disciplina, este grupo profesional realizó algunas vinculaciones con las prácticas de enseñanza y aprendizaje. Estas categorías se presentaron en la zona de la Periferia o en la Zona de contraste, lo que implicaría que son considerados como elementos más flexibles y sujetos a modificaciones según las circunstancias. En cambio, en los estudios restantes, al ser consultados sobre la Docencia y la Escuela, los docentes muestran en el núcleo de la representación una fuerte presencia de la importancia atribuida a estas actividades.

Podemos pensar que aunque las demandas sociales ejercen una fuerte presión sobre los docentes e intentan desviarlos en su desempeño profesional hacia otras funciones (por ejemplo, asistenciales, sanitarias o de contención social), éstas no constituyen la esencia de la función social que los docentes se auto atribuyen y pretenden conservar a lo largo del tiempo.

La similitud entre las estructuras de los distintos grupos de docentes nos muestran que existe una representación que va más allá de las diferencias -en la experiencia, en el nivel educativo en el que trabajan, en las diversas formaciones disciplinares de base, e inclusive, en 
los diferentes contextos en que se desempeñan- que permite describir lo que son y lo que hacen los docentes, correspondiéndose con los postulados educativos tradicionales, marcando las diferencias con otros grupos y otras actividades profesionales.

\section{Referencias}

Abric, Jean Claude. (2001). Prácticas sociales y representaciones. México: Ed. Coyoacán.

Álvarez Méndez, Juan Manuel. (2001). Entender la didáctica, entender el currículum. Madrid, España: Miño y Dávila.

Braslavsky, Cecilia y Birgin, Alejandra (Ed.) (1992). Formación de profesores: Impacto, pasado y presente. Bs. As, Argentina: Editorial Miño y Dávila S.A.

Brígido, Ana María. (2006). Sociología de la educación: temas y perspectivas fundamentales. Córdoba -Argentina: Editorial Brujas

Camilloni, Alicia. (2007). El saber didáctico. Bs. As., Argentina: Paidós.

Chacoma, Monica, Mazzitelli, Claudia y Aparicio, Miriam. (2009, agosto). La escuela y su representación social: una mirada desde alumnos urbanos y urbanos-marginales. Ponencia presentada y publicada en las Memorias del III Congreso Internacional de Educación, Santa Fe, Argentina.

Graca, Margarida; Moreira, Marco Antonio y Caballero, Concesa. (2004). Representacoes sobre a Matemática, seu ensino e aprendizagem: um estudo exploratório. Revista Investigacoes em Ensino de Ciencias, 9, (1). Recuperado el 10 de febrero de 2005 de http://www.if.ufrgs.br/ienci/

Jodelet, Denise. (1986). La Representación social: fenómenos, concepto y teoría. En Serge Moscovici (Ed.), Psicología social, II (pp. 469-494). Barcelona- España: Ed. Paidós.

Kaplan, Carina. (2003). Buenos y malos alumnos. Descripciones que predicen. Bs. As., Argentina: Aique.

Lacolla, Liliana. (2005). Representaciones sociales: una manera de entender las ideas de nuestros alumnos. Revista Electrónica de la Red de Investigación Educativa 1, (3). Recuperado el 3 de marzo de 2008 de http://revista.iered.org.

Litwin, Edith. (1998). La investigación didáctica en un debate contemporáneo. En Mario Carretero, José Antonio Castorina y Ricardo Baquero (Ed.), El debate constructivista (pp. 162-190). Bs. As., Argentina: Aique. 
López Alonso, Alfredo y Stefani, Dorina. (2005). Representaciones sociales de la vida: su variación a través del género y la edad de las personas. Su convergencia y divergencia. Revista Signos Universitarios, XXIV (41), 23-118.

Marín Martinez, Nicolás (2003). Conocimientos que interaccionan en la enseñanza de las ciencias. Enseñanza de las Ciencias, 21 (1), 65-78.

Martínez Filomeno, Sandra. (2003). La representación social de la profesión docente: creencias respecto de la ciencia y la enseñanza. Comunicación publicada en las Memorias de las $1^{\circ}$ Jornadas sobre Representaciones Sociales, Investigación y Práctica. Bs.As. - Argentina.

Mazzitelli, Claudia. (2007). El aprendizaje de la Física como reelaboración conceptual a la luz de algunas teorías psicosociales. Tesis Doctoral en Educación, Universidad Nacional de Cuyo, Argentina.

Mazzitelli, Claudia y Aparicio, Miriam. (2007, abril). Identificando las representaciones sociales de docentes sobre las Ciencias Naturales. Ponencia presentada y publicada en las Memorias del IV Congreso Nacional y II Internacional de Investigación Educativa (Universidad del Comahue), Cipolletti, Argentina.

Mazzitelli, Claudia; Aguilar, Susana; Guirado, Ana y Olivera, Adela. (2009, agosto). El lugar de la didáctica en las representaciones sociales de los docentes de Ciencias. Ponencia presentada y publicada en las Memorias del III Congreso Internacional de Educación, Santa Fe- Argentina.

Mazzitelli, Claudia; Aguilar, Susana; Guirado, Ana y Olivera, Adela. (2009). Representaciones sociales de los profesores sobre la docencia: contenido y estructura. Revista Educación, Lectura y Sociedad, 6 (6), 265-290.

Mellado, Vicente. 2000. ¿Es adecuada la formación científica del profesorado de ciencias de secundaria para sus necesidades profesionales? Alambique, (24), 57-65.

Mellado Jiménez, Vicente; Blanco Nieto, Lorenzo y Ruiz Macías, Constantino. (1999). Aprender a enseñar ciencias experimentales en la formación inicial del profesorado: estudios de caso sobre la enseñanza de la energía. España: Universidad de Extremadura.

Mora, Martín. (2002). La teoría de las Representaciones Sociales de Serge Moscovici. Revista Athenea Digital, (2). Recuperado el 10 de febrero de 2005 de www.bib.uab.es/pub/athenea

Moscovici, Serge. (1961/1979). El Psicoanálisis, su imagen y su público. Bs. As., Argentina: Ed. Huemul.

Nuñez, Graciela; Pereira, Raúl; Maturano, Carla y Mazzitelli, Claudia. (2007, mayo). Dificultades en la formación disciplinar de docentes de Ciencias Naturales. Ponencia presentada y publicada en las Memorias de las I Jornadas Nacionales de Investigación 
Educativa y II Jornadas Regionales de Investigación Educativa (Universidad Nacional de Cuyo), Mendoza, Argentina.

Petracci, Mónica y Kornblit, Ana Lía. (2007). Representaciones sociales: una teoría metodológicamente pluralista. En Ana Lía Kornblit (Ed.), Metodologías cualitativas en Ciencias Sociales (pp. 91-111). Bs.As., Argentina: Editorial Biblos.

Saura Llamas, Octavio \& De Pro Bueno, Antonio. (2000). La enseñanza y el aprendizaje del conocimiento físico. En Perales Palacios, Francisco y Cañal de León, Pedro (Ed.) Didáctica de las Ciencias Experimentales (pp. 389-419). Alcoy, España: Marfil.

Tenti Fanfani, Emilio. (2007). La Escuela y la cuestión social. Ensayos de sociología de la educación. Bs. As, Argentina: Siglo veintiuno Editores.

Vaillant, Denise. (2007). La identidad docente. Conferencia en I Congreso Internacional "Nuevas Tendencias en la Formación Permanente del Profesorado" GTD-PREAL-ORT.

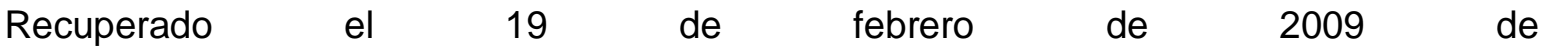
http://161.116.7.34/congresformacio/conferenciesvi.htm

Valles, Miguel. (2000). Técnicas cualitativas de investigación social. Reflexión metodológica y práctica profesional. Madrid, España: Ed. Síntesis.

Welti, Reinaldo. (2002). Concepciones de estudiantes y profesores acerca de la energía de las ondas. Enseñanza de las Ciencias, 20 (2), 261-270. 